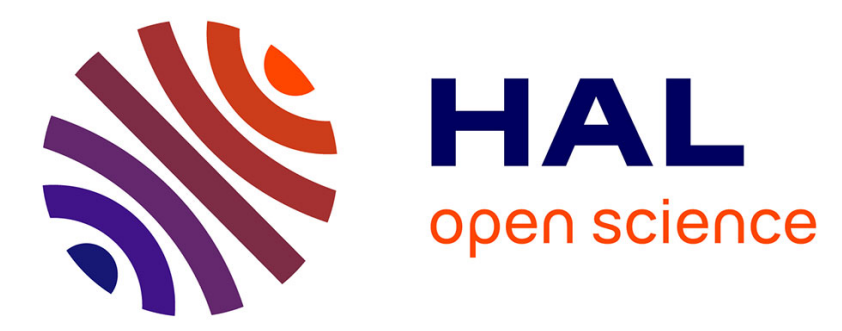

\title{
Simulation of laser-sensor digitizing for on-machine part inspection
}

\author{
Nguyen Duy Minh Phan, Yann Quinsat, Claire Lartigue
}

\section{To cite this version:}

Nguyen Duy Minh Phan, Yann Quinsat, Claire Lartigue. Simulation of laser-sensor digitizing for on-machine part inspection. International Joint Conference on Mechanics, Design Engineering \& Advanced Manufacturing, Sep 2016, Catane, Italy. hal-01358951

\section{HAL Id: hal-01358951 \\ https://hal.science/hal-01358951}

Submitted on 1 Sep 2016

HAL is a multi-disciplinary open access archive for the deposit and dissemination of scientific research documents, whether they are published or not. The documents may come from teaching and research institutions in France or abroad, or from public or private research centers.
L'archive ouverte pluridisciplinaire HAL, est destinée au dépôt et à la diffusion de documents scientifiques de niveau recherche, publiés ou non, émanant des établissements d'enseignement et de recherche français ou étrangers, des laboratoires publics ou privés. 


\title{
Simulation of laser-sensor digitizing for on- machine part inspection
}

\author{
Nguyen Duy Minh PHAN, Yann QUINSAT and Claire LARTIGUE ${ }^{1 *}$ \\ ${ }^{1}$ LURPA, ENS Cachan, Univ. Paris-Sud, Université Paris-Saclay, 94235 Cachan, France \\ * Corresponding author. Tel.: +33-147-402-986 ; fax: +33-147-402-200. E-mail address: \\ lartigue@lurpa.ens-cachan.fr
}

\begin{abstract}
Integrating measurement operations for on-machine inspection in a 5axis machine tool is a complex activity requiring a significant limitation of measurement time in order not to penalize the production time. When using a laserplane sensor, time optimization must be done while keeping the quality of the acquired data. In this paper, a simulation tool is proposed to assess a given digitizing trajectory. This tool is based on the analysis of sensor configurations relatively to the geometry of the studied part.
\end{abstract}

Keywords: In situ measurement, Laser plane sensor, Digitizing quality, Trajectory simulation

\section{Introduction}

Integrating inspection procedures within the production process involves rapid decision-making regarding the conformity of parts. In the case of on-machine inspection for instance, part geometry measurements are performed in the same phase as the machining operations without removing the part from its set-up, which facilitates comparing the machined part to its $\mathrm{CAD}$ model for the conformity analysis. This also contributes to reducing the time allocated to measurement. Within this context, a few recent studies have focused on the use of laser-plane sensors to carry out on-machine inspections as they have a great ability to measure deviations of machining parts within a time consistent with on-machine inspection $[1,2]$. In the particular case of the milling process, laser-plane sensors can be integrated in the machine tool, the sensor replacing the cutting tool. As the measurement operation is performed while the process is stopped, one of the main issues concerns sensor path-planning. In fact, the time allocated to measurement must be minimized to preserve global production time, but the quality of the acquired data must be sufficient to measure potential deviations. 


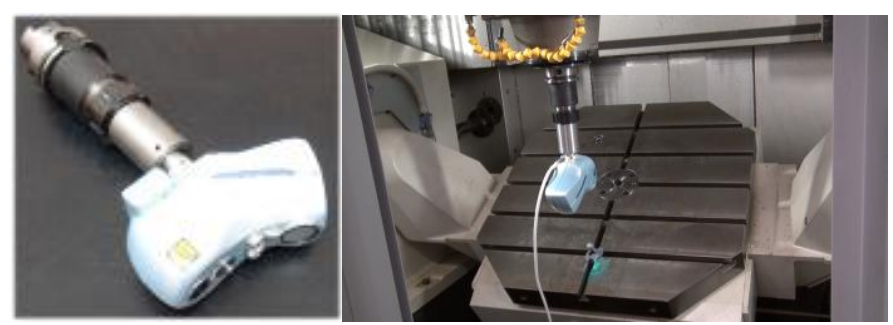

Fig. 1. Laser-sensor in the machine-tool

The integration of a laser-sensor in a machine-tool is an issue little addressed in the literature. The fact that the sensor accessibility is increased, considering the 5 degrees of freedom plus the spindle rotation, is an opportunity. Indeed, as for laser mounted on industrial robots, this gives the sensor the possibility to scan an object from any direction even along curved paths [3]. Wu et al. [4] propose a method of sensor path planning for surface inspection on a 6 degree-of-freedom robot that automatically adapts its trajectory to the complex shape of the object by continuously changing the viewing direction of the scanner mounted on the robot. Each viewpoint of the planned path must however satisfy several constraints: field of view, scanning distance, view angle and overlap. Within the context of sensor path planning for part inspection, it is quite classical to impose some constraints to the sensor relatively to the part to be measured. In [5], authors introduce the concept of visibility (local and global) to generate a sensor trajectory well-adapted to the control of complex parts. Prieto et al. propose to keep the sensor as normal as possible to the surface, while obeying a criterion of quality depending on both the scanning distance and the sensor view angle [6]. Son et al. include additional constraints such as the number of required scans and the checking of occlusions [7]. Yang and Ciarallo use a genetic algorithm to obtain a set of viewing domains and a list of observable entities for which the errors are within an acceptable tolerance [8]. The approach developed in [9] relies on the representation of the part surface as a voxel map, for which the size of each voxel is defined according to the sensor field of view (fov). To each voxel, a unique point of view is associated in function of visibility and quality criteria leading to a set of admissible viewpoints to ensure the surface digitizing with a given quality. Mavrinac et al. [10] formalize the search of sensor viewpoints under constraints for $3 \mathrm{D}$ inspection using an active triangulation system. Each viewpoint (sensor configuration) is assessed thanks to a performance function that results from the combination of constraint functions to be respected. This interesting approach seems valuable to assess the validity of a sensor trajectory prior to its optimization.

This paper deals with laser-sensor trajectories well-adapted for on-machine inspection on a 5 -axis milling machine-tool. First, we propose to define an original description format of the sensor trajectory directly interpretable by the CNC of the machine-tool. The laser-plane sensor takes the place of the cutting tool in the spindle. This original format takes advantage of the additional degree of freedom 
given by the spindle rotation. The sensor trajectory is a series of ordered viewpoints that must satisfy a set of constraints (visibility, quality, number of viewpoints, overlaps, etc.). Prior to the stage of trajectory optimization, it might be interesting to have a tool assessing given trajectories according to the constraints to be satisfied. In this direction, the second part of the paper presents a method for simulating the digitizing from a given trajectory.

\section{Sensor trajectory in 5-axes}

A description format must be defined that can be interpretable by the $\mathrm{CNC}$ of the machine-tool. The study can be applied for laser-plane sensor types for which the field of view (fov) is planar (2D) (figure 2). The sensor trajectory consists in a set of ordered sensor configurations (each configuration defining a viewpoint), i.e. a set of positions and orientations. The sensor orientation is given by the couple of vectors: $\vec{v}_{C}$, the director vector of light-beam axis, and $\vec{v}_{L}$, the director vector of the digitizing line (figure 2). By analogy of cutting tool trajectories for which the cutter location point (CL point) is the tool extremity [11], the sensor position is defined through the point $\mathrm{C}_{\mathrm{E}}$ which positions the digitizing line: $\overrightarrow{C_{0} C_{E}}=d * \cdot \vec{v}_{c}$. Therefore, in the part frame, the sensor trajectory is a set of configurations $\left(C_{E} ; \vec{v}_{C} ; \vec{v}_{L}\right)$ expressed in the part frame as a set of coordinates $\left(X, Y, Z, I, J, K, I^{*}\right.$, $J^{*}, K^{*}$ ) (figure 2). This trajectory is expressed in the machine-tool frame thanks to the Inverse Kinematics Transform, leading to $(X, Y, Z, A, C, W)$ where $A$ and $C$ are the classical angles for a RRTTT machine tool and $W$ allows the spindle indexation. This additional degree of freedom is particularly interesting to orient the laser beam relatively to the surface.
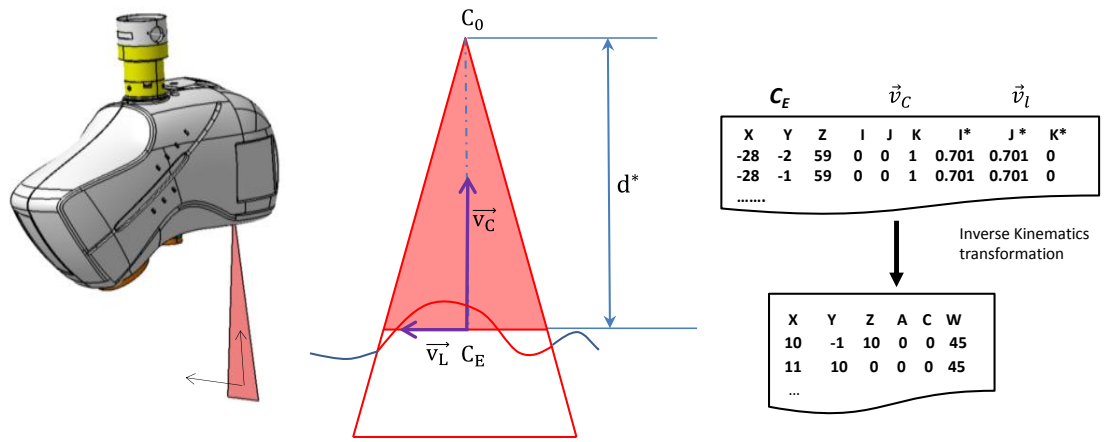

Fig. 2. Parameters defining the sensor trajectory for on-machine inspection

Indeed, the sensor trajectory is classically defined to satisfy a set of constraints, generally visibility and quality constraints, leading to the ordered series of sensor 
configurations $\left(C_{E} ; \vec{v}_{C}\right)$. The width of the scanning line, which can be assimilated to the width of the cutting tool, varies in function of the scanning distance $d^{*}$ (see figure 2). The additional degree of freedom given by the spindle indexation $W$ permits to orient $\vec{v}_{L}$. It could thus be possible to plan a sensor trajectory so that the width of the scanning line is maximized by modifying the sensor indexation.

After describing the parameter setting for the definition of a digitizing trajectory on a 5-axis CNC machine-tool, a simulation tool is proposed with the aim of assessing the quality of the acquired data.

\section{Digitizing simulation}

Considering a given sensor trajectory, a simulator has been developed to assess this trajectory with regard to a set of constraints. As reported in the literature, the most usual constraints are visibility, and digitizing quality. The laser scanner is characterized by its actual fov, which corresponds to the area of the scanning plane which is visible by the camera. The fov is thus defined by its height, $\mathrm{H}$, and its widths $\mathrm{L}_{\min }$ and $\mathrm{L}_{\max }$, each width corresponding to the minimal and maximal height in the fov. Hence, a portion of part surface is visible if it belongs to the fov so defined (figure 4). However, as the sensor moves from one configuration $\left(C_{E i} ; \vec{v}_{C i}\right)$ to another $\left(C_{E i+1} ; \vec{v}_{C i+1}\right)$, a portion of surface is digitized if it belongs to the swept volume created by the displacement of the laser plane from the first configuration to the second, as displayed in figure 4.

The orientation of the sensor relatively to the surface and the digitizing distance characterize the quality of the acquired data. Therefore, the digitizing quality is evaluated according to both the scanning distance $d$, and the view angle, $\alpha$ as we proposed in previous studies $[5,13]$. Parameters defining the digitizing conditions are summarized in figure 4 and table1.

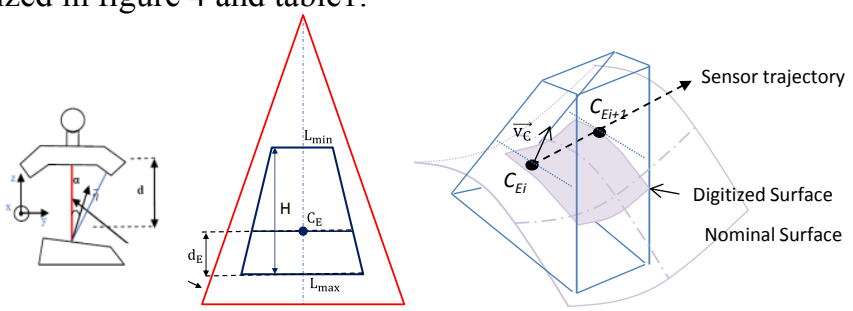

Fig. 4. Parameters of the laser sensor and digitizing volume between 2 configurations

To develop our approach, we take advantage of the formalism proposed in $[4,10]$, in which the constraints to be verified are expressed as a combination of functions. Such formalism is rather flexible as it permits to add or remove constraints in function of the complexity we want to introduce in trajectory generation. The CAD model of the part is tessellated through a STL format. This gives a 
mesh defined by a set $S_{T}$ of $\mathrm{n}$ triangular facets $T_{j}$. Each facet $T_{j}$ is defined by 3 vertices denoted $V_{j}^{i}$, and the normal vector to the facet is denoted $\vec{n}_{j}$ (see table 1).

Table 1. Parameters for surface digitizing

\begin{tabular}{ll}
\hline Mesh Parameters & Digitizing parameters \\
\hline$S_{T}$, set of n triangular facets & $V_{C_{E i} C_{E i+1}}$, swept volume between 2 configurations \\
$T_{j}$, facet $\mathrm{j}, T_{j} \in S_{T}, j \in[1, n]$ & $d_{E}$, distance from the bottom to $C_{E i}$ in the fov \\
$S_{v}$, set of vertices & $H$, distance from the bottom to the top in the fov \\
$V_{j}^{k}$, vertex of a facet $T_{j}, k \in[1,3]$ & $\alpha_{\max }$, limited view angle of the sensor \\
$\vec{n}_{j}$, normal to the facet $T_{j}$ & $d_{V_{j}^{k}, \text { distance from the bottom of } f o v \text { to the vertex } V_{j}^{k}}$ \\
\hline
\end{tabular}

In a first approach only 2 functions are considered: the visibility function and the quality function. These functions are applied to the tessellated model.

\subsection{Visibility function}

The visibility function is used to determine the facets which we denote as seen by the laser sensor. As the trajectory is a set of sensor configurations, the visibility function is defined for each trajectory segment, i.e. between two successive configurations. For each facet $T_{j}$ of the CAD model, the function is defined as a combination of two functions as expressed in equation 1 :

$$
F_{V}^{*}\left(T_{j}\right)=F_{V}\left(T_{j}\right) \cdot F_{s \alpha}\left(T_{j}\right)
$$

The swept facet function $F_{V}\left(T_{j}\right)$ checks if the facet belongs to the volume swept by the laser beam between 2 configurations $V_{C_{E i}} C_{E i+1}$. A facet belongs to the swept volume if all its vertices belong to the swept volume:

$$
F_{V}\left(T_{j}\right)= \begin{cases}1, & \text { if } \forall k \in[1,3], V_{j}^{k} \in V_{C_{E i} C_{E i+1}} \\ 0, & \text { otherwise }\end{cases}
$$

Generally, the view angle is limited [12]. If the angle between the normal vector to the facet and $\vec{v}_{C}$ exceeds the maximal view angle $\alpha_{\max }$, the facet is not seen. This is expressed by:

$$
F_{s \alpha}\left(T_{j}\right)= \begin{cases}1, & \text { if } \vec{n}_{j} \cdot \vec{v}_{c} \geq \cos \left(\alpha_{\text {max }}\right) \\ 0, & \text { otherwise }\end{cases}
$$


At the end of this stage, when the whole sensor trajectory is considered (i.e. when all the trajectory segments defined by $\left(C_{E i} ; \vec{v}_{C i}\right)\left(C_{E i+1} ; \vec{v}_{C i+1}\right)$ are considered), all the facets verifying $F_{V}^{*}\left(T_{j}\right)=1$ are characterized as seen and define the set $S_{T}^{S}=\left\{T_{j} \in S_{T}, F_{V}^{*}\left(T_{j}\right)=1\right\}$.

\subsection{Quality function}

The visibility of the facet does not ensure the digitizing quality. Indeed, numerous studies point out the importance of the digitizing distance and of the view angle on the digitizing noise, factors that strongly influence the digitizing quality [5, $9,12,13]$. Quality is ensured when the digitizing noise is lesser than a threshold, threshold generally given by the user in function of the considered application. This involves admissible ranges for both the digitizing distance and the view angle allowing the definition of the quality function as follows:

$$
F_{w s}\left(T_{j}\right)=F_{V}^{*}\left(T_{j}\right) \cdot F_{w s d}\left(T_{j}\right) \cdot F_{w s \alpha}\left(T_{j}\right)
$$

In equation (4), $F_{w s d}$ and $F_{w s}$ account for the quality in terms of digitizing distance and view angle respectively. Therefore, a facet is said well-seen in terms of digitizing distance if all its vertices belong to the admissible range of digitizing distances $I_{a d}$ :

$$
F_{w s d}\left(T_{j}\right)= \begin{cases}1, & \text { if } \forall k \in[1,3], d_{V_{j}^{k}} \in I_{a d} \\ 0, & \text { otherwise }\end{cases}
$$

A facet is said well-seen in terms of view angle, if the angle between the normal vector to the facet and $\vec{v}_{C}$, belongs to the admissible range of view angles defined by $\alpha_{1}$ and $\alpha_{2}$ :

$$
F_{w s \alpha}\left(T_{j}\right)= \begin{cases}1, & \text { if } \cos \left(\alpha_{1}\right) \leq \vec{n}_{j} \cdot \vec{v}_{c} \leq \cos \left(\alpha_{2}\right) \\ 0, & \text { otherwise }\end{cases}
$$

At the end, all the facets verifying $F_{w s}\left(T_{j}\right)=1$ are characterized as well-seen and define the set $S_{T}^{w S}=\left\{T_{j} \in S_{T}^{S}, F_{w s}\left(T_{j}\right)=1\right\}$. All the other seen facets are tagged as poorly-seen and in turn define a set $S_{T}^{p s}=\left\{T_{j} \in S_{T}^{S}, F_{w s}\left(T_{j}\right)=0\right\}$ with $S_{T}^{S}=S_{T}^{w s} \cup S_{T}^{p s}$. The facets which are not-seen define the set $S_{T}^{n s}$, complementary of $S_{T}^{S}$ in $S_{T}: S_{T}=S_{T}^{S} \cup S_{T}^{n s}$. 


\section{Results and discussion}

The objective is here to validate our simulator by comparing digitizing obtained using our simulator to the actual digitizing, and considering various trajectories. The simulator is tested using a case study, and for the laser sensor Zephyr KZ25 (www.kreon3d.com). Although most of the sensor parameters are given by the manufacturer, a protocol of sensor qualification is required to identify the actual sensor parameters such as the dimensions of the fov, or the limited view angle, but also to identify quality parameters that define the admissible ranges of digitizing distances and view angles.

\subsection{Sensor parameters}

First, the dimensions of the fov are identified by simply measuring a reference plane. As the intersection of the reference plane and the laser-beam is a line, the height $H$ of the fov is identified by observing if the line is visible in the CCD. The experiment gives $H=50 \mathrm{~mm}$. According to the protocol defined in [13], the evolution of the digitized noise, denoted $\delta$, is identified in function of the digitizing distance and the view angle. The digitizing noise accounts for the dispersion of the measured points with respect to a reference element, and it is usually evaluated by measuring a reference plane surface for different digitizing distances and various view angles.
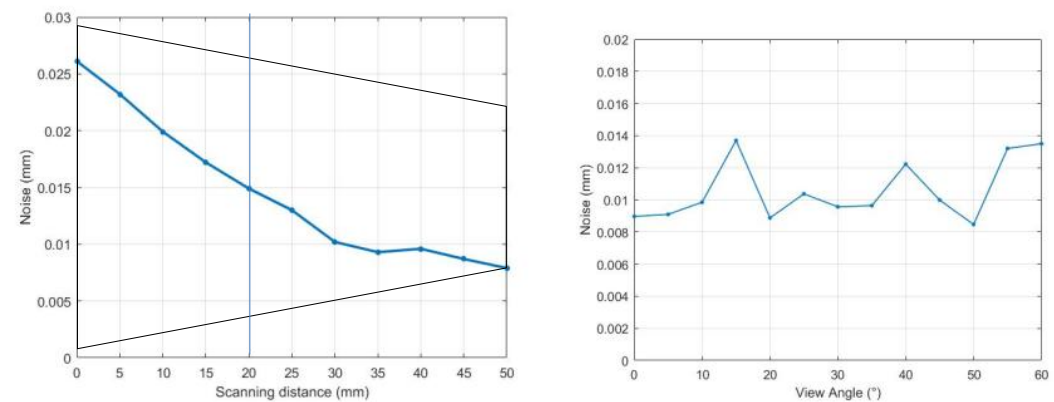

Fig. 5. Noise in function of the scanning distance (a) and the view angle (b).

The evolution of the digitizing noise in function of the digitizing distance exhibits a significant decrease of the noise from the bottom position to the top position in the fov (figure 5a). On the other hand, the evolution of the noise in function of the view angle does not show a significant trend (figure $5 b$ ). However, it can be pointed out that the maximal view angle is equal to $\alpha_{\max }=60^{\circ}$, and that for the whole range $\left[0^{\circ} ; 60^{\circ}\right]$, the noise remains lesser than $0.015 \mathrm{~mm}$. Considering that value as the threshold $\delta_{a d}$ for quality, the admissible range of digitizing distances 
$I_{a d}$ is defined by $[20 ; 50] \mathrm{mm}$. Those two intervals guarantee a digitizing with a noise lesser than $\delta_{a d}=0.015 \mathrm{~mm}$.

\subsection{Simulator tests}

The sensor trajectories used to test our simulator are classical pocket-type trajectories. For the first tests, the sensor orientation is constant, and the trajectory consists in a set of points $C_{E}$ defined at a constant altitude z (figure 6). To assess the simulator, the simulated digitizing is compared to the actual one. For this purpose, actual digitizing was carried out using a Coordinate Measuring Machine (CMM) equipped with a motorized indexing head, which enables the scanner to be oriented according to repeatable discrete orientations. We choose to assess our simulator using a CMM, because a 3-axis Cartesian CMM is a machine with less geometrical defaults than a machine-tool. But this does not change anything in the principle of our simulator. On the CMM, the orientations of the sensor are given by the two rotational angles A and B. Therefore, the trajectories expressed in the part coordinate system (for the simulation) must first be expressed in the CMM coordinate system (figure 6).

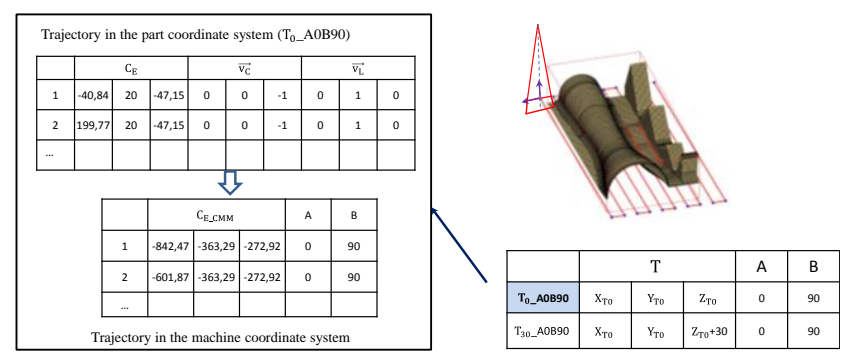

Fig. 6. Scanning trajectories for test $\left(\mathrm{A}=0^{\circ} ; \mathrm{B}=90^{\circ}, \mathrm{z}=0 \mathrm{~mm}\right)$.

Different trajectories for various digitizing distances and sensor orientations have been tested. Only results associated with one orientation $\left(\mathrm{A}=0^{\circ} ; \mathrm{B}=90^{\circ}\right)$ and two different distances $(\mathrm{z}=0$ and $\mathrm{z}=30 \mathrm{~mm})$ are commented in this paper. The algorithm is applied to the tessellated CAD model of the part, and facets are classified in the corresponding set according to visibility and quality functions as proposed in section 3. To simplify the representation, a color code is adopted: well-seen facets are green, poorly-seen facets are orange, and not-seen facets are red (table 2). On the other hand, the actual digitizing gives a point cloud which is registered onto the mesh model. For each facet, a cylinder, whose basis is the triangle defining the facet and the height is the maximal measurement error, is created. 
Table 2. Results for actual and simulated digitizing.

\begin{tabular}{l}
\hline Simulation of the digitizing Actual digitizing \\
$\begin{array}{l}\mathrm{A}=0^{\circ} ; \mathrm{B}=90^{\circ} ; \\
\mathrm{z}=0 \mathrm{~mm}\end{array}$ \\
$\mathrm{~A}=0^{\circ} ; \mathrm{B}=90^{\circ} ;$ \\
$\mathrm{z}=30 \mathrm{~mm}$
\end{tabular}

The set of digitized points belonging to the cylinder so defined corresponds to the actual digitized facet. To compare actual digitizing to its simulation, we have to characterize each facet according to visibility and quality functions in the same way. In this direction, we consider that a facet is not-seen if the density of points associated with the facet is less than 5 points $/ \mathrm{mm}^{2}$; the facet color is red. For each facet, the geometrical deviations between the digitized points and the facet are calculated. The associated standard deviation accounts for the actual digitizing noise. If the noise is greater than the threshold $\delta_{a d}=0.015 \mathrm{~mm}$, the facet is tagged as poorly-seen, and its color is set to orange. Conversely, if the noise is lesser than $\delta_{a d}$, the facet is tagged as well-seen, and its color is green. Results displayed in table 2 bring out the good similarity between simulation and actual digitizing. This is particularly marked for the trajectory $z=0$. However, some differences exist for which the simulator underestimates the digitizing. A whole area which appears red in the simulation is green in the actual digitizing (on the left of the part for the trajectory $\mathrm{z}=30 \mathrm{~mm}$ for instance). This is likely due to the fact that the digitizing noise is evaluated using an artefact with a specific surface treatment which makes the surface very absorbing, whereas the part is coated with a white powder that matifies the surface. Digitizing is thus facilitated. Nevertheless, the simulator turns out to be an interesting predictive tool prior to sensor trajectory planning.

\section{Conclusion}

Within the context of on-machine inspection using laser-plane digitizing systems, sensor trajectory planning is a challenge. To ensure the efficiency of the measurement, it is necessary to minimize measurement time while ensuring the 
quality of the acquired data. The presented work proposes a description format of a sensor well-adapted to on-machine inspection on 5-axis machine-tools. Given a digitizing trajectory, a simulation tool of the acquired data quality is presented. After a real digitizing, a good similarity between simulation and actual digitizing can be observed. The simulator is thus an interesting predictive tool that can be used to assist in finding the best strategy to digitize the part with a quality consistent with geometrical deviations obtained in milling.

\section{References}

1. L. Dubreuil, Y. Quinsat, C. Lartigue, Multi-sensor approach for multi-scale machining defect detection, Joint Conference On Mechanical, June 2014, Toulouse, France, Research in Interactive Design Vol. 4

2. F. Poulhaon, A. Leygue, M. Rauch, J-Y. Hascoet, and F. Chinesta, Simulation-based adaptative toolpath generation in milling processes, Int. J. Machining and Machinability of Materials, 2014, $15(3 / 4)$, pp.263-284.

3. S. Larsson and Johan AP Kjellander. Path planning for laser scanning with an industrial robot. Robotics and Autonomous Systems, 2008, 56(7), pp.615-624.

4. Q. $\mathrm{Wu}, \mathrm{J} . \mathrm{Lu}, \mathrm{W}$. Zou, and D. Xu. Path planning for surface inspection on a robot-based scanning system. In Mechatronics and Automation (ICMA), IEEE International Conference on, 2015, pp. 2284-2289.

5. A. Bernard, M. Véron, Visibility theory applied to automatic control of $3 \mathrm{~d}$ complex parts using plane laser sensors. CIRP Annals-Manufacturing Technology, 2000, 49(1), pp.113-118.

6. Prieto, F., Redarce, H., Lepage, R., and Boulanger, P., Range image accuracy improvement by acquisition planning. In Proceedings of the 12th conference on vision interface (VI'99), Trois Rivieres, Québec, Canada, 1999, pp.18-21.

7. Son, S., Park, H., and Lee, K. H., Automated laser scanning system for reverse engineering and inspection. International Journal of Machine Tools and Manufacture, 2002, 42(8), pp.889-897.

8. Yang, C. C. and Ciarallo, F. W., Optimized sensor placement for active visual inspection. Journal of Robotic Systems, 2001, 18(1), pp.1-15.

9. Lartigue, C., Quinsat, Y., Mehdi-Souzani, C., Zuquete-Guarato, A., and Tabibian, S., Voxelbased path planning for $3 \mathrm{~d}$ scanning of mechanical parts. Computer-Aided Design and Applications, 2014, 11(2), pp.220-227.

10. Mavrinac, A., Chen, X., and Alarcon-Herrera, J. L., Semiautomatic model-based view planning for active triangulation 3-d inspection systems. Mechatronics, IEEE/ASME Transactions on, 2015, 20(2), pp.799-811.

11. S. Lavernhe, Y. Quinsat, C. Lartigue, Model for the prediction of 3D surface topography in 5-axis milling, International Journal of Advanced Manufacturing Technology, 2010, 51, pp. 915-924.

12. M. Mahmud, D. Joannic, M. Roy, A. Isheila, J.-F. Fontaine, 3D part inspection path planning of a laser scanner with control on the uncertainty, Computer-Aided Design , 2011, 43, pp. $345-355$.

13. C. Mehdi-Souzani, Y. Quinsat, C. Lartigue, P. Bourdet' A knowledge database of qualified digitizing systems for the selection of the best system according to the application, CIRP Journal of Manufacturing Science and Technology, 2016, DOI: doi:10.1016/j.cirpj.2015.12.002 